\title{
C2 Gene
}

National Cancer Institute

\section{Source}

National Cancer Institute. C2 Gene. NCI Thesaurus. Code C126956.

This gene is involved in the classical pathway of the complement system. 